\title{
Implications of Nerve Fiber Density on the Diagnosis and Treatment of Juvenile Fibromyalgia
}

\author{
Nabeel Ahmed (D)', Marie Vigouroux $\mathbb{D}^{2,3}$, Pablo Ingelmo $\mathbb{D}^{3-5}$ \\ 'Faculty of Medicine, McGill University, Montreal, QC, Canada; ${ }^{2}$ Faculty of Dentistry, McGill University, Montreal, QC, Canada; ${ }^{3}$ Edwards Family \\ Interdisciplinary Center for Complex Pain, Montreal Children's Hospital, Montreal, QC, Canada; ${ }^{4}$ Research Institute, McGill University Health \\ Centre, Montreal, QC, Canada; ${ }^{5}$ Alan Edwards Research Center for Pain, McGill University, Montreal, QC, Canada
}

Correspondence: Marie Vigouroux, Family Interdisciplinary Center for Complex Pain, Montreal Children's Hospital, I00I boul. Décarie A02.3523, Montreal, QC, H4A 3JI, Canada, Tel +I 5 I4 4I2 4448, Fax +I 5 I4 4I2 434I, Email Marie.vigouroux@mail.mcgill.ca

\begin{abstract}
Juvenile fibromyalgia (JFM) is a condition that presents as chronic widespread musculoskeletal pain and affects children and adolescents. JFM remains a challenging diagnosis, as it is both based on subjective criteria and the pathogenesis is poorly understood. Small fiber neuropathy (SFN) is a distinct condition, which is characterized by pathology of small A-delta and C fibers, and can present similarly to JFM. Small fiber pathology is characterized by reduced intraepidermal nerve fiber density (IENFD) on skin biopsy. Recent studies have found that as many as half of patients with JFM can demonstrate decreased IENFD, in pattern similar to SFN. This phenomenon has been referred to as small fiber pathology. The meaning of these findings was disputed; however, the current consensus remains that fibromyalgia and SFN are distinct conditions. Additionally, among patients with fibromyalgia, there are two phenotypes: those with small fiber pathology and those without. The purpose of this review was to characterize the role assessment of IENFD plays in the clinical context. We conducted a narrative review of pertinent articles pertaining to JFM, SFN and small fiber pathology in fibromyalgia. We concluded that assessment of IENFD should be completed if SFN is suspected either when a patient first presents or in patients who were previously diagnosed with fibromyalgia and SFN is later suspected. Distinguishing between JFM and SFN is important because recommended therapies differ between the two conditions. However, there is no evidence to support the use of skin biopsy to distinguish between the two discussed fibromyalgia phenotypes. More studies are needed to elucidate whether IENFD varies with morbidity and if both fibromyalgia phenotypes vary in their response to different therapeutic regimens.
\end{abstract}

Keywords: small fiber neuropathy, skin biopsy, chronic pain, pediatric

\section{Introduction}

Juvenile fibromyalgia (JFM) is a chronic pain condition causing significant morbidity affecting children and adolescents. The prevalence of JFM amongst school-aged children lies in the range of 2 to $6 \%$ and found most commonly in female adolescents. $^{1}$

JFM as a diagnosis remains difficult for clinicians and patients alike due to its syndromic nature. Given its characterization as an ensemble of non-specific symptoms, current guidelines recommend diagnosis if patients exhibit a threshold number of symptoms..$^{2-4}$ The mechanisms responsible for pain in affected patients are not well elucidated.

The findings of recent studies have raised the question of the utility of skin biopsy to assess nerve fiber density in fibromyalgia patients. In studies in which patients with clinical diagnoses of fibromyalgia underwent skin biopsy, abnormality in small nerve fibers was detected in roughly half of cases. ${ }^{5-7}$ Interestingly, these findings are reminiscent of a distinct condition, small fiber neuropathy (SFN), which is characterized by pathology of small fibers, specifically the degeneration of thinly myelinated type A-delta and unmyelinated $\mathrm{C}$ fibers, which can be associated with clinical findings characteristic of fibromyalgia in addition to neuropathic pain and disturbances. ${ }^{6,8}$

SFN as a disorder has only been characterized in pediatric patients quite recently; epidemiologic data is thus unavailable; however, there is evidence that this condition exists among school-aged children with widespread pain; 
some of these patients had previously been diagnosed with fibromyalgia and others with phenomena including acute monophasic erythromelalgia, central sensitization, pain-amplification syndrome and chronic fatigue. ${ }^{9,10}$

Assessment of nerve fiber density is the standard of care for small fiber neuropathy in adults. ${ }^{11}$ It can become a useful tool in the diagnosis of this condition in children.

The aim of this paper is to characterize the indication for skin biopsy to assess nerve fiber density in pediatric patients with presumed JFM in clinical practice. In order to do so, we conducted a narrative review of JFM, SFN and small fiber pathology in fibromyalgia. The majority of the existing literature describing the latter two only describes the adult population.

\section{Juvenile Fibromyalgia \\ Presentation}

JFM presents as chronic widespread musculoskeletal pain. This pain is generally diffuse; however, patients can have socalled tender points or specific painful bodily locations, usually several. ${ }^{3,12}$ Symptomatology also includes fatigue. ${ }^{3}$ It can be associated with other clinical entities including migraine, irritable bowel syndrome, and depression. ${ }^{13}$

\section{Diagnosis}

The literature outlining diagnostic criteria for JFM is limited. When the Yunus and Masi criteria was published in 1985, it was the first guideline for the diagnosis of fibromyalgia in the pediatric population and remains widely used. ${ }^{12}$ More recently, the 2010 American College of Rheumatology (ACR) Adult Fibromyalgia Criteria was evaluated for use in female pediatric patients. ${ }^{3}$

Under both criteria, diagnosis is symptom-based. The Yunus and Masi criteria stipulates that in order to make a diagnosis of JFM, patients must exhibit generalized musculoskeletal pain at three or more sites, five or more "tender points", and three other minor criteria; four tender points and five minor criteria are also sufficient. ${ }^{12}$ The 2010 ACR criteria provides a scoring metric, by which patients are assigned a numerical value based on the number of painful bodily locations and associated symptoms; diagnosis is indicated at a threshold score. ${ }^{3}$ The 2010 ACR criteria does away with the tender point exam, which is often criticized as it may be done incorrectly or omitted in clinical practice. ${ }^{3}$ Notably, between both criteria, there are no objective measures to diagnose or assess the severity of the condition.

\section{Treatment}

Evidence-based treatment of JFM is predominantly non-pharmacologic. Recommended treatments include exercise and physical therapy; however, adherence can be challenging for patients in the long term. ${ }^{14}$ Additionally, cognitive behavioral therapy has been shown to be effective in the treatment of JFM. ${ }^{14}$

The evidence regarding the efficacy of pharmacologic treatment is generally poor. ${ }^{15}$ There is some evidence that the use of serotonin-norepinephrine reuptake inhibitors and selective serotonin reuptake inhibitors can be effective in pediatric patients. ${ }^{14}$ In adults, tricyclic antidepressants and anti-convulsant drugs such as gabapentin and pregabalin have been used; however, there is no strong evidence for these being used successfully in pediatric patients. ${ }^{14,15}$ Pregabalin specifically was found to not improve morbidity in a statistically significant manner in pediatric patients. ${ }^{16}$ Pharmacologic therapy should thus be used sparingly due to lack of evidence regarding efficacy and known adverse effects.

\section{Prognosis}

The prognosis of JFM seems to be highly variable, with certain studies indicating a positive prognosis, and more recent data showing that fibromyalgia symptoms among adolescents tend to persist into adulthood. ${ }^{17,18}$ Physical therapy, exercise-based therapy and cognitive behavioral therapy have been found to reduce symptoms in the short term, but their long-term effects on prognosis are unclear. ${ }^{1,14} \mathrm{~A}$ large-scale trial investigating the combination of these modalities is currently underway. ${ }^{19}$ 


\section{Small Fiber Neuropathy}

SFN is a clinical condition characterized by pathology of small fibers, specifically the degeneration of thinly myelinated type A-delta and unmyelinated $\mathrm{C}$ fibers, which is associated with neuropathic pain and disturbances. ${ }^{8}$ Prevalence amongst adult populations has been characterized in The Netherlands to be 52.95 (95\% CI 42.47-65.23) cases/ $100,000 .^{20} \mathrm{SFN}$ as a disorder has only been characterized in pediatric patients recently; although there is evidence that this condition exists among school-aged children, epidemiologic data and extensive literature pertaining to this population are unavailable. ${ }^{9,10}$ As such, the literature reviewed pertaining to SFN largely describes the condition in adults.

\section{Presentation}

Patients with SFN can present with clinical findings characteristic of fibromyalgia; however, SFN is a distinct condition. ${ }^{6}$ Patients with SFN can present with chronic pain, which is typically symmetric and length dependent, contrary to fibromyalgia. ${ }^{7,21}$ Lodahl et al have also characterized that certain symptoms such as paresthesia and peripheral autonomic dysfunction are more common in patients with SFN. ${ }^{22}$ SFN can be primary and idiopathic but can also be secondary to diabetes, connective tissue disorders, viral infection, autoinflammatory syndromes, and neurotoxin exposure. ${ }^{23,24}$

\section{Diagnosis}

In adults, two sets of criteria have been proposed for the diagnosis of SFN, the Besta Criteria (Devigili et al 2008) and the NEURODIAB Criteria (Tesfaye et al 2010). ${ }^{25}$ The Besta Criteria for diagnosis of SFN is met if patients exhibit two of the following: clinical signs of small fiber impairment, abnormal cold and warm thresholds on foot on quantitative sensory testing (QST), or reduced intraepidermal nerve fiber density (IENFD). ${ }^{26}$ The NEURODIAB Criteria was proposed in diabetic patients, grading patients as possibly, probably or definitely having SFN based on how many of the following are met: clinical signs of small fiber damage, normal sural nerve conduction study, abnormal QST, or reduced IENFD. ${ }^{27}$ The Besta Criteria was reappraised and validated in 2019 using the NEURODIAB Criteria as gold standard, and strict agreement was found between the two. ${ }^{25}$

Furthermore, Devigili et al establish that assessment of IENFD is the most reliable tool for diagnosing SFN, followed by warm and cold QST thresholds. ${ }^{25}$ Skin biopsy to assess IENFD constitutes a Level A recommendation from the European Federation of Neurological Societies for the diagnosis of SFN. ${ }^{11}$ This technique has been used to diagnose SFN in several pediatric patients, with age-adjusted norms; however, no protocol has been established for diagnosis of SFN in this population. ${ }^{9,10,28}$

SFN diagnostic protocol also incorporates the use of QST. Patients with SFN can exhibit abnormal cold and warm detection thresholds. ${ }^{25}$ It should be noted that patients with fibromyalgia can also demonstrate similar abnormal QST findings. ${ }^{7}$

\section{Treatment}

Pharmacologic treatment of SFN can be etiology-specific when there is a suspected cause. For instance, in immunemediated causes, such as Sjogren's syndrome and systemic lupus erythematosus, IVIg has been shown to improve morbidity in pediatric and adult patient populations. ${ }^{8,29}$ In SFN associated with inflammatory conditions, corticosteroids have also been shown to be useful in adults and children. ${ }^{29}$ Additionally, in patients with sarcoidosis, IVIg and anti-TNF therapy are reportedly beneficial. ${ }^{8}$

Finally, in adult patients with idiopathic SFN, tricyclic antidepressants, serotonin-norepinephrine reuptake inhibitors, and anticonvulsants, such as pregabalin and gabapentin, have been shown to relieve pain. ${ }^{8,24}$

\section{Prognosis}

In adults, SFN has been shown to generally remain a stable condition. ${ }^{30}$ In the case of idiopathic SFN, prognosis varies based on individual response to pharmacologic therapy, which can be effective. ${ }^{24}$ In children, immune-mediated and inflammatory SFN seem to be amenable to disease-modifying pharmacologic therapy including IVIg and corticosteroids. ${ }^{9,31}$ IVIg, however, has not been found to be useful in the cases of idiopathic SFN in adults. ${ }^{32}$ 


\section{Evidence of Small Fiber Pathology in Patients with Fibromyalgia Syndrome}

The skin biopsies of a significant portion of adults previously diagnosed with fibromyalgia exhibit small fiber pathology. The common characteristic was the reduction in the epidermal nerve fiber density. ${ }^{6,7}$ Oaklander et al suggested that those findings were consistent with the diagnosis of SFN and that certain patients diagnosed with fibromyalgia were in fact misdiagnosed. ${ }^{6}$ Fibromyalgia is a clinical diagnosis and a diagnosis of exclusion; if patients meet the criteria for small fiber neuropathy, that is the diagnosis. Üçeyler et al specified, however, that fibromyalgia still exists as a distinct condition and that patients with fibromyalgia can be grouped into those with small fiber pathology and those without. ${ }^{31}$

A meta-analysis of eight studies showed the random effects prevalence of small fiber pathology in adults with fibromyalgia to be $49 \%$ (95\% CI: 38 to $60 \%) .{ }^{33}$ In a case-control study including 15 pediatric patients, Boneparth et al found that 53\% (95\% CI: 26 to 79\%) of patients with fibromyalgia had reduced IENFD. ${ }^{5}$

Evdokimov et al found some degree of denervation in $63 \%$ of 117 adult female patients with fibromyalgia. Patients with denervation of both the lower leg and upper thigh presented a more severe fibromyalgia phenotype. ${ }^{34}$ Fasolino et al studied 57 patients (54 females), 30\% of whom presented small fiber pathology. However, there were no differences between patients with and without small fiber pathology in the clinical evaluation, QST, or laser-evoked potentials. ${ }^{35}$

Elucidation of the mechanism for denervation as well as its potential causative effect on pain is still under debate. For instance, Leinders et al has found a correlation between the expression of miR-let-7d and small fiber pathology in patients with fibromyalgia. ${ }^{36}$ Evdokimov et al found a differential expression of pain mediators and axon pathfinders in the skin cells of patients with fibromyalgia, however with no perceived correlation to decreased IENFD. ${ }^{37}$ Even if some evidence suggested a pathologic relationship between intraepidermal nerve denervation and clinical presentation in patients with fibromyalgia syndrome, it cannot be said that the denervation observed in certain patients with fibromyalgia is the cause of their pain. Proving this relationship is important because, while fibromyalgia is generally considered to cause nociplastic pain, pain in patients with fibromyalgia and small fiber pathology could be characterized as neuropathic pain. ${ }^{38,39}$ Correct classification is important because it can impact treatment and prognosis.

\section{Is It Possible to Establish a Differential Diagnosis with Skin Biopsies?}

Fibromyalgia and SFN can both present with similar symptoms; however, it is possible to distinguish between the two as long as guidelines are followed. ${ }^{21,35}$ Distinguishing between fibromyalgia and SFN is relevant as certain pharmacologic therapy is indicated in SFN and not fibromyalgia. ${ }^{2,24}$ A comparison of the two conditions is shown in Table 1.

The differential diagnosis of patients with fibromyalgia is quite extensive, and many other disorders can also be comorbid with this syndrome. Key disorders to consider may be those with rheumatologic, infectious, endocrine, or neurologic origin or primary musculoskeletal pain, the diagnostic protocols for which are beyond the scope of this paper. ${ }^{4,12}$

Skin biopsy constitutes the standard of care for the diagnosis of SFN and should be used as a diagnostic tool in cases where this entity is suspected. ${ }^{26,27}$ However, it should not be used on all patients with chronic pain, not to weaken its diagnostic power.

Half of pediatric patients with fibromyalgia may present small fiber pathology. ${ }^{5}$ However, the assessment of IENFD in pediatric patients with fibromyalgia is rarely used in clinical practice.

Hypothetically, if patients clinically diagnosed with fibromyalgia were to undergo skin biopsy, two groups of patients would emerge: those with fibromyalgia without small fiber pathology and patients with fibromyalgia with small fiber pathology. It is possible that these two subgroups would respond differently to different treatment modalities. However, this hypothesis would need to be explored further.

The diagnosis of fibromyalgia syndrome in pediatric patients can be made using the 2010 ACR Criteria adapted for use in pediatric patients. Assessment of IENFD, either by skin biopsy or by an analogous test, is clinically indicated if SFN is suspected either when a patient first presents or in patients who were previously diagnosed with fibromyalgia, and SFN is later suspected. Patients with presumed JFM refractory to non-pharmacologic therapy may indeed have SFN. ${ }^{6}$ Investigation of this entity is warranted prior to starting pharmacologic therapy, which is not well supported by evidence in the case of JFM. A flow diagram of a proposed diagnostic approach to JFM and SFN is depicted in Figure 1. 
Table I Comparison of Juvenile Fibromyalgia and Small Fiber Neuropathy

\begin{tabular}{|c|c|c|}
\hline & Juvenile Fibromyalgia & Small Fiber Neuropathy \\
\hline Symptoms & $\begin{array}{l}\text { - Widespread musculoskeletal pain } \\
\text { - Diffuse, with tender points or specific painful locations } \\
\text { - Chronicity } \\
\text { - Fatigue } \\
\text { - Associated conditions include: IBS, migraine, depression } \\
\text { - Hyperalgesia and allodynia to tactile and thermal stimuli } \\
\text { - Autonomic dysfunction }\end{array}$ & $\begin{array}{l}\text { - Pain, paresthesia and/or peripheral autonomic dysfunction } \\
\text { - Often symmetric and may be length-dependent } \\
\text { orders, diabetes, viral infection, autoinflammatory syndromes } \\
\text { and neurotoxin exposure }\end{array}$ \\
\hline $\begin{array}{l}\text { Available } \\
\text { tests }\end{array}$ & Clinical diagnosis & $\begin{array}{ll}\text { - } & \text { Skin biopsy - assessment of IENFD } \\
\text { - } & \text { QST } \\
\text { - } & \text { QSART }\end{array}$ \\
\hline $\begin{array}{l}2010 \\
\text { Modified } \\
\text { ACR Criteria }\end{array}$ & $\begin{array}{l}\text { 20I0 Modified ACR Criteria } \\
\text { - WPI: score based on number of painful bodily locations ( } 19 \\
\text { total) } \\
\text { - SS: score based on number of symptoms including fatigue, } \\
\text { waking feeling tired, and concentration/memory problems } \\
\text { - Fibromyalgia if WPI } \geq 7+\text { SS } \geq 5 \text { OR WPI 3-6+SS } \geq 9\end{array}$ & $\begin{array}{l}\text { No established pediatric criteria Besta Criteria (adult): SFN if } \geq 2 \\
\text { of the following } \\
\text { - Clinical signs of small fiber impairment (pinprick and thermal } \\
\text { sensory loss and/or allodynia and/or hyperalgesia) } \\
\text { - Skin biopsy (abnormal IENFD) } \\
\text { - QST (abnormal warm and cold thresholds) }\end{array}$ \\
\hline $\begin{array}{l}\text { Treatment } \\
\text { options }\end{array}$ & $\begin{array}{l}\text { Strong evidence: } \\
\text { - Non-pharmacologic } \\
\circ \text { Exercise } \\
\circ \text { Physical therapy } \\
\circ \text { Cognitive behavioral therapySome evidence: } \\
\text { - Pharmacologic } \\
\circ \text { Serotonin-norepinephrine reuptake inhibitors } \\
\text { - Selective serotonin reuptake inhibitors } \\
\text { Some evidence in adults: } \\
\text { - Pharmacologic } \\
\text { O Pregabalin }{ }^{\mathrm{a}} \text { \& gabapentin } \\
\text { - Tricyclic antidepressants }\end{array}$ & $\begin{array}{l}\text { Etiology specific (if known): } \\
\text { - Immune mediated (ex: Sjogren's, SLE): IVlg } \\
\text { - Inflammatory: corticosteroids }{ }^{\mathrm{b}} \\
\text { - Sarcoidosis: IVlg, anti-TNF } \\
\text { Idiopathic - Evidence in adults: } \\
\text { - Pharmacologic } \\
\circ \text { Tricyclic antidepressants } \\
\circ \text { Serotonin-norepinephrine reuptake inhibitors } \\
\circ \text { Anticonvulsants (pregabalin, gabapentin) }\end{array}$ \\
\hline Prognosis & $\begin{array}{l}\text { - Variable: can improve or chronic symptoms can continue } \\
\text { into adulthood } \\
\text { - Non-pharmacologic therapy has short-term benefits } \\
\text { - Still an area of ongoing research }\end{array}$ & $\begin{array}{l}\text { - Good prognosis in immune-mediated and inflammatory SFN } \\
\text { with disease-modifying treatment } \\
\text { - In adults, disease remains stable and pain can be controlled } \\
\text { pharmacologically } \\
\text { - Further research needed in children }\end{array}$ \\
\hline
\end{tabular}

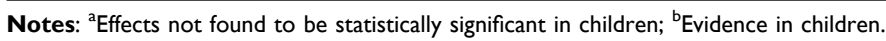

Abbreviations: IBS, irritable bowel syndrome; ACR, American College of Rheumatology; WPI, widespread pain index; SS, symptom severity; IENFD, intraepidermal nerve fiber density; QST, quantitative sensory testing; QSART, quantitative sudomotor axon reflex test; SLE, systemic lupus erythematosus; IVIg, intravenous immunoglobulin; TNF, tumor necrosis factor; SFN, small fiber neuropathy.

\section{Moving Forward}

While SFN is often idiopathic, it has also been characterized as secondary to metabolic or infectious causes, connective tissue disorders, neurotoxin exposures, and inherited neuropathies. ${ }^{23,24}$ In children, it has also been associated with autoinflammatory syndromes. ${ }^{40}$ Fibromyalgia, on the other hand, has been conventionally considered as a primary idiopathic syndrome, often associated with other clinical entities such as irritable bowel syndrome, migraine headaches, and depression. ${ }^{13}$

Further research should aim to elucidate whether the conditions associated with having fibromyalgia and small fiber pathology are more closely aligned with disorders thought to be causative of SFN, compared to patients without small fiber pathology. 


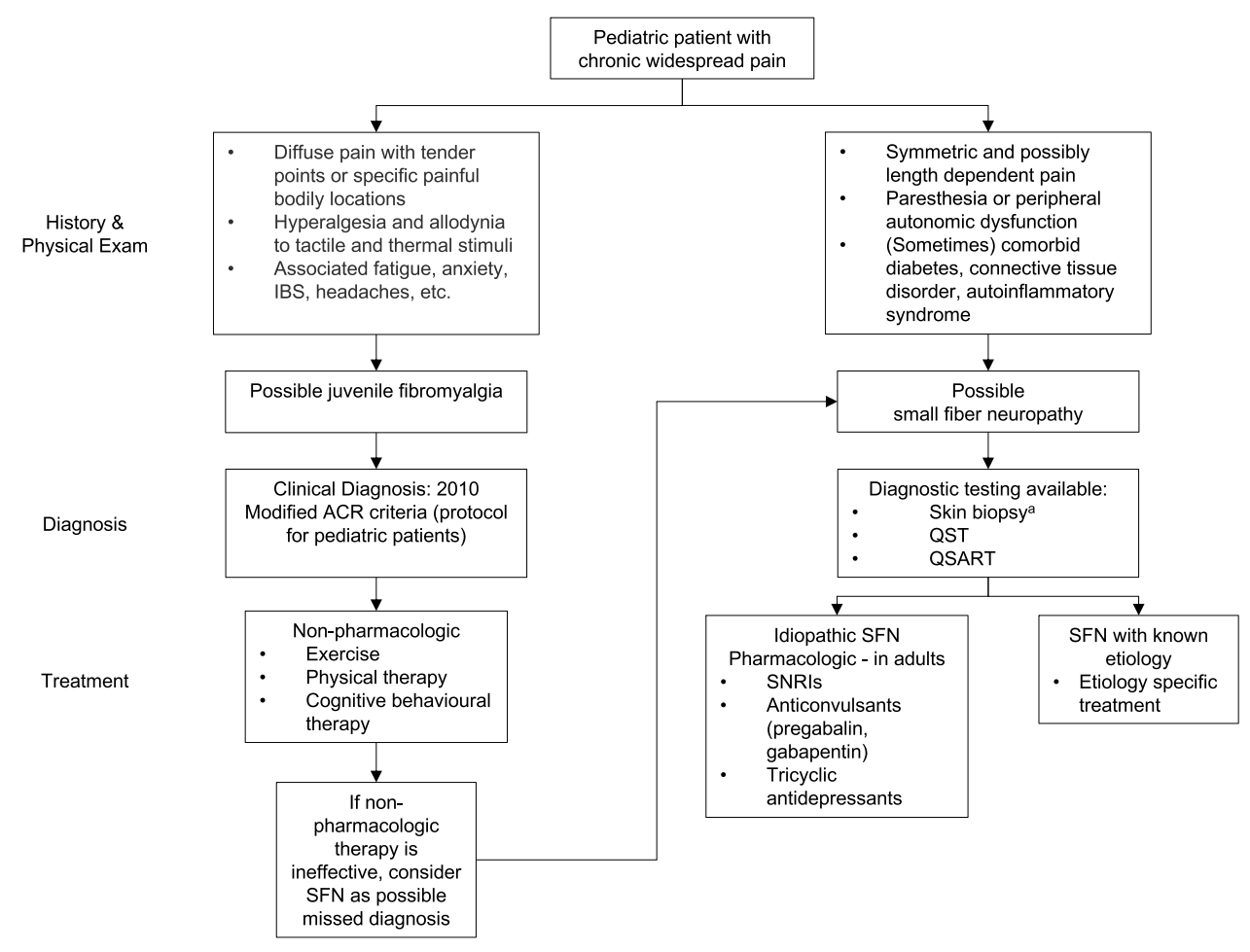

Figure I Proposed diagnostic approach to the investigation of JFM and SFN.

Note: a: Gold standard.

Abbreviations: IBS, irritable bowel syndrome; ACR, American College of Rheumatology; SFN, small fiber neuropathy; QST, quantitative sensory testing; QSART, quantitative sudomotor axon reflex test; SNRI, serotonin-norepinephrine reuptake inhibitor.

The characterization of the clinical utility of IENFD in pediatric patients with fibromyalgia remains limited. Another avenue for further research would be to elucidate whether patients with fibromyalgia with and without small fiber pathology respond differently to therapeutic strategies.

There is also conflicting research regarding the clinical significance of small fiber pathology amongst adult patients with fibromyalgia as it pertains to the burden of disease. ${ }^{34,35}$ More data is likely needed to conclusively determine an association. As much of the current data is cross-sectional, a longitudinal prospective study may be better suited to prospectively characterize how an individual patient's IENFD varies with morbidity. The results of such investigation could elucidate the possibility of tracking disease progression in a quantitative prognostic manner. As there is evidence that corneal denervation parallels intraepidermal denervation, a corneal confocal microscopy could potentially be used as a surrogate for skin biopsy as a minimally invasive procedure for such monitoring. ${ }^{34}$

\section{Conclusion}

In summary, JFM is a chronic pain condition that is predominantly idiopathic and syndromic in nature. Recent research has uncovered the existence of pathology involving small nerve fibers, in a pattern resembling SFN. ${ }^{5-7}$

The clinical significance and pathogenesis of these findings are currently ambiguous and deserve to be elucidated. However, it is important to be aware of these two distinct conditions and their diagnostic modalities, as the treatment and prognosis are different.

Broadly, if a pediatric patient presents with chronic pain and a symptom profile suggestive of small fiber neuropathy, skin biopsy is indicated for the diagnosis of this condition. Additionally, in patients previously diagnosed with fibromyalgia which is refractory to non-pharmacologic therapy, it may be useful to consider SFN as a possibly missed diagnosis prior to beginning pharmacologic treatment.

\section{Disclosure}

The authors report no conflicts of interest in this work. 


\section{References}

1. Kashikar-Zuck S, Ting TV. Juvenile fibromyalgia: current status of research and future developments. Nat Rev Rheumatol. 2014;10(2):89-96. doi:10.1038/nrrheum.2013.177

2. Farhad K, Oaklander AL. Fibromyalgia and small-fiber polyneuropathy: what's in a name? Muscle Nerve. 2018;58(November):611-613. doi:10.1002/mus.26179

3. Ting TV, Barnett K, Lynch-Jordan A, Whitacre C, Henrickson M, Kashikar-Zuck S. 2010 American College of Rheumatology Adult Fibromyalgia criteria for use in an adolescent female population with juvenile fibromyalgia. J Pediatr. 2016;169:181-188. doi:10.1016/j.jpeds.2015.10.011

4. Arnold LM, Bennett RM, Crofford LJ, et al. AAPT diagnostic criteria for fibromyalgia. J Pain. 2019;20(6):611-628. doi:10.1016/j. jpain.2018.10.008

5. Boneparth A, Chen S, Horton DB, et al. Epidermal neurite density in skin biopsies from patients with juvenile fibromyalgia. $J$ Rheumatol. 2020;13 (3):287-288. doi:10.3899/jrheum.200378

6. Oaklander AL, Herzog ZD, Downs HM, Klein MM. Objective evidence that small-fiber polyneuropathy underlies some illnesses currently labeled as fibromyalgia. Pain. 2013;154(11):2310-2316. doi:10.1016/j.pain.2013.06.001

7. Üçeyler N, Zeller D, Kahn AK, et al. Small fibre pathology in patients with fibromyalgia syndrome. Brain. 2013;136(6):1857-1867. doi:10.1093/ brain/awt053

8. Basantsova NY, Starshinova AA, Dori A, Zinchenko YS, Yablonskiy PK, Shoenfeld Y. Small-fiber neuropathy definition, diagnosis, and treatment. Neurol Sci. 2019;40(7):1343-1350. doi:10.1007/s10072-019-03871-x

9. Faignart N, Nguyen K, Soroken C, et al. Acute monophasic erythromelalgia pain in five children diagnosed as small-fiber neuropathy. Eur J Paediatr Neurol. 2020;28:198-204. doi:10.1016/j.ejpn.2020.06.004

10. Oaklander AL, Klein MM. Evidence of small-fiber polyneuropathy in unexplained, juvenile-onset, widespread pain syndromes. Pediatrics. 2013;131(4):e1091-e1100. doi:10.1542/peds.2012-2597

11. Lauria G, Hsieh ST, Johansson O, et al. European Federation of Neurological Societies/Peripheral Nerve Society guideline on the use of skin biopsy in the diagnosis of small fiber neuropathy. Report of a joint task force of the European Federation of Neurological Societies and the Peripheral Nerve Society. Eur J Neurol. 2010;17(7):903-e49. doi:10.1111/j.1468-1331.2010.03023.x

12. Yunus MB, Masi AT. Juvenile primary fibromyalgia syndrome. A clinical study of thirty-three patients and matched normal controls. Arthritis Rheum. 1985;28:138-145. doi:10.1002/art.1780280205

13. Bennett RM, Friend R. Secondary fibromyalgia. J Rheumatol. 2019;46(2):127-129. doi:10.3899/jrheum.180611

14. Coles ML, Uziel Y. Juvenile primary fibromyalgia syndrome: a review - treatment and prognosis. Pediatr Rheumatol. 2021;19:1-10.

15. Eccleston C, Fisher E, Cooper TE, et al. Pharmacological interventions for chronic pain in children: an overview of systematic reviews. Pain. 2019;160(8):1698-1707. doi:10.1097/j.pain.0000000000001609

16. Arnold LM, Schikler KN, Bateman L, et al. Safety and efficacy of pregabalin in adolescents with fibromyalgia: a randomized, double-blind, placebo-controlled trial and a 6-month open-label extension study. Pediatr Rheumatol. 2016;14(1):1-11. doi:10.1186/s12969-016-0106-4

17. de Sanctis V, Abbasciano V, Soliman AT, et al. The juvenile fibromyalgia syndrome (JFMS): a poorly defined disorder. Acta Biomed. 2019;90 (1):134-148. doi:10.23750/abm.v90i1.8141

18. Kashikar-Zuck S, Cunningham N, Peugh J, et al. Long-term outcomes of adolescents with juvenile-onset fibromyalgia into adulthood and impact of depressive symptoms on functioning over time. Pain. 2019;160(2):433-441. doi:10.1097/j.pain.0000000000001415

19. Kashikar-Zuck S, Briggs MS, Bout-Tabaku S, et al. Randomized clinical trial of Fibromyalgia Integrative Training (FIT teens) for adolescents with juvenile fibromyalgia - study design and protocol. Contemp Clin Trials. 2021;103(January):106321. doi:10.1016/j.cct.2021.106321

20. Peters MJH, Bakkers M, Merkies ISJ, Hoeijmakers JGJ, van Raak EPM, Faber CG. Incidence and prevalence of small-fiber neuropathy: a survey in the Netherlands. Neurology. 2013;81(15):1356-1360. doi:10.1212/WNL.0b013e3182a8236e

21. Terkelsen AJ, Karlsson P, Lauria G, Freeman R, Finnerup NB, Jensen TS. The diagnostic challenge of small fibre neuropathy: clinical presentations, evaluations, and causes. Lancet Neurol. 2017;16(11):934-944. doi:10.1016/S1474-4422(17)30329-0

22. Lodahl M, Treister R, Oaklander AL. Specific symptoms may discriminate between fibromyalgia patients with vs without objective test evidence of small-fiber polyneuropathy. Pain Rep. 2018;3(1):e633. doi:10.1097/PR9.0000000000000633

23. Freeman R, Gewandter JS, Faber CG, et al. Idiopathic distal sensory polyneuropathy: ACTTION diagnostic criteria. Neurology. 2020;95:1005-1014. doi:10.1212/wn1.0000000000010988

24. Sène D. Small fiber neuropathy: diagnosis, causes, and treatment. Jt Bone Spine. 2018;85(5):553-559. doi:10.1016/j.jbspin.2017.11.002

25. Devigili G, Rinaldo S, Lombardi R, et al. Diagnostic criteria for small fibre neuropathy in clinical practice and research. Brain. 2019;142 (12):3728-3736. doi:10.1093/brain/awz333

26. Devigili G, Tugnoli V, Penza P, et al. The diagnostic criteria for small fibre neuropathy: from symptoms to neuropathology. Brain. 2008;131 (7):1912-1925. doi:10.1093/brain/awn093

27. Tesfaye S, Boulton AJM, Dyck PJ, et al. Diabetic neuropathies: update on definitions, diagnostic criteria, estimation of severity, and treatments. Diabetes Care. 2010;33(10):2285-2293. doi:10.2337/dc10-1303

28. Hoeijmakers JGJ, Faber CG, Miedema CJ, Merkies ISJ, Vles JSH. Small fiber neuropathy in children: two case reports illustrating the importance of recognition. Pediatrics. 2016;138(4). doi:10.1542/peds.2016-1215

29. Oaklander AL, Nolano M. Scientific advances in and clinical approaches to small-fiber polyneuropathy: a review. JAMA Neurol. 2019;76 (10):1240-1251. doi:10.1001/jamaneurol.2019.2917

30. MacDonald S, Sharma TL, Li J, Polston D, Li Y. Longitudinal follow-up of biopsy-proven small fiber neuropathy. Muscle Nerve. 2019;60 (4):376-381. doi:10.1002/mus.26648

31. Üçeyler N, Sommer C. Objective evidence that small-fiber polyneuropathy underlies some illnesses currently labeled as fibromyalgia. Pain. 2013;154(11):2569. doi:10.1016/j.pain.2013.06.037

32. Geerts M, de Greef BTA, Sopacua M, et al. Intravenous immunoglobulin therapy in patients with painful idiopathic small fiber neuropathy. Neurology. 2021;96(20):e2534-e2545. doi:10.1212/WNL.0000000000011919

33. Grayston R, Czanner G, Elhadd K, et al. A systematic review and meta-analysis of the prevalence of small fiber pathology in fibromyalgia: implications for a new paradigm in fibromyalgia etiopathogenesis. Semin Arthritis Rheum. 2019;48:933-940. doi:10.1016/j.semarthrit.2018.08.003 
34. Evdokimov D, Frank J, Klitsch A, et al. Reduction of skin innervation is associated with a severe fibromyalgia phenotype. Ann Neurol. 2019;86 (4):504-516. doi:10.1002/ana.25565

35. Fasolino A, Di Stefano G, Leone C, et al. Small-fibre pathology has no impact on somatosensory system function in patients with fibromyalgia. Pain. 2020;161(10):2385-2393. doi:10.1097/j.pain.0000000000001920

36. Leinders M, Doppler K, Klein T, et al. Increased cutaneous miR-let-7d expression correlates with small nerve fiber pathology in patients with fibromyalgia syndrome. Pain. 2016;157(11):2493-2503. doi:10.1097/j.pain.0000000000000668

37. Evdokimov D, Kreß L, Dinkel P, et al. Pain-associated mediators and axon pathfinders in fibromyalgia skin cells. $J$ Rheumatol $J$ Rheumatol. 2020;47:140-148. doi:10.3899/jrheum.190248

38. Finnerup NB, Haroutounian S, Kamerman P, et al. Neuropathic pain: an updated grading system for research and clinical practice. Pain. 2016;157 (8):1599-1606. doi:10.1097/j.pain.0000000000000492

39. Fitzcharles MA, Cohen SP, Clauw DJ, Littlejohn G, Usui C, Häuser W. Nociplastic pain: towards an understanding of prevalent pain conditions. Lancet. 2021;397(10289):2098-2110. doi:10.1016/S0140-6736(21)00392-5

40. Shinkarevsky Fleitman I, Nevo Y, Harel L, et al. Small-fiber neuropathy associated with autoinflammatory syndromes in children and adolescents. Muscle Nerve. 2020;61(6):791-796. doi:10.1002/mus.26857

Journal of Pain Research

\section{Publish your work in this journal}

The Journal of Pain Research is an international, peer reviewed, open access, online journal that welcomes laboratory and clinical findings in the fields of pain research and the prevention and management of pain. Original research, reviews, symposium reports, hypothesis formation and commentaries are all considered for publication. The manuscript management system is completely online and includes a very quick and fair peer-review system, which is all easy to use. Visit http://www.dovepress.com/testimonials.php to read real quotes from published authors.

Submit your manuscript here: https://www.dovepress.com/journal-of-pain-research-journal 